\title{
Tipología o hermenéutica ¿Tensión fenomenológica en las dos fases de juicio arendtiano?
}

\author{
Typology or hermeneutics. Phenomenological tension in
}

the two phases of Arendtian judgment?

JULIÁN GARCÍA LABRADOR ${ }^{\mathrm{a}}$

\section{Resumen}

La muerte sorprendió a Arendt sin que pudiera terminar la última parte de La Vida del Espíritu, dedicada al juicio. Ello ha propiciado que, sin una posición definitiva, los interpretes de Arendt hayan dinstinguido dos fases de su teoría del juicio y hayan tratado de explicar la relación entre ambas. La primera fase estaría centrada en el actor, mientras que la segunda refleja la postura del espectador de la acción. Este artículo aborda la cuestión desde una posición fenomenológica, advirtiendo que estas dos fases revelan la tensión del giro hermenéutico de la fenomenología: o bien admitimos la posibilidad de una hermenéutica sin fin para facilitar el ejercicio político del actor, o bien, establecemos ciertos ejemplares de juicio con los que identificar la realidad política, sin poder ir más allá de lo que hemos establecido como tipología.

Palabras clave: Arendt. Fenomenología. Juicio. Hermenéutica. Tipología.

\section{Abstract}

Arendt died without finishing the last part of The Life of the Mind, dedicated to judgment. Without a definitive position, Arendt's interpreters have divided two phases of their theory of judgment and have tried to explain the relationship between the two. The first phase

a Universidad Rey Juan Carlos. Madrid, España. Doctor en Filosofía, e-mail: julian.labrador@urjc.es 
would be centered on the actor, while the second one reflects the viewer's position of action. This article approaches the question from a phenomenological position, noting that these two phases reveal the tension of the hermeneutic turn of phenomenology: either we admit the possibility of an endless hermeneutic to facilitate the political exercise of the actor, or, we establish certain exemplary of judgment with which to identify the political reality, without going beyond what we have established as a typology.

Keywords: Arendt. Phenomenology. Judgment. Hermeneutics. Typology.

\section{Introducción}

Pensadora independiente y no sistemática (Taylor, 2002; López, 2001), Arendt trata de presentar lo político en el mundo contemporáneo y su relación con las facultades espirituales. recuperando el juicio político de la mano de Kant. Se trata de una recuperación problemática, porque Arendt asume la distinción actor / espectador del juicio reflexionante kantiano, sin asumir su origen (la filosofía del derecho de Kant), es decir, sin presentar el juicio en un horizonte trascendental. Ello ha motivado que algunas veces Arendt sitúe el juicio en el ámbito del actor y que en otras ocasiones lo haga en el del espectador. Advirtiendo esta disyuntiva, muchos de los intérpretes coindicen en señalar que existen dos etapas bastante claras en el tratamiento arendtiano del juicio. Otros advierten una continuidad de fondo y una tensión que no acaba de romperse. Según Ronald Beiner (2003) y Richard Bernstein (1986) habría que distinguir dos fases. En un primer momento, para Arendt el juicio uniría su suerte al ejercicio político, como si se tratara de una preparación para la nueva acción. En una segunda etapa, el juicio descifra el significado de las acciones que ya han tenido lugar. En el primer momento, el juicio es central para el actor; en el segundo, es el espectador quien se sirve del juicio. La primera etapa incluye los escritos donde Arendt trata de encontrar una forma de cognición ligada a la acción (Arendt va tanteando su descripción fenoménica, nunca completada), mientas la segunda tiene lugar a partir de 1971, cuando, tras la controversia sobre Eichmann y el debate sobre la responsabilidad (Arendt, 2007a), adopta el juicio reflexionante kantiano como modelo de juicio histórico. Michael Denneny (1979) sostiene, sin embargo, que sería difícil encontrar una 
segunda fase definida por el carácter contemplativo, puesto que en La Vida del Espiritu la voluntad está ligada a la acción política y no a la contemplación (Gray, 1979; Jacobitti, 1988). Steinberger (1990) sigue a Denneny para indicar que no podemos perder de vista que el juicio es en Arendt un asunto eminentemente político y, por tanto, nunca podremos concebirlo ni siquiera en su segunda fase, en oposición a la vida pública. Juzgar es "la forma que el pensamiento adquiere en el mundo político" (Steinberger, 1990: 812). De igual manera Diana Taylor (2002) considera que la segunda etapa del juicio en Arendt no puede desligarse de este carácter político.

El núcleo del problema es cómo vincular las facultades espirituales con las actividades del mundo, lo cual se hace conflictivo por la separación que Arendt establece entre política y filosofía. En la primera fase, el juicio, posibilita la nueva acción política, tras el desastre totalitario, al ir de la mano de la comprensión. Así, comprender el mundo es condición para que la libertad pueda ser ejercida de nuevo, una vez que el régimen de terror y la ideología totalitaria han cercenado la espontaneidad de los seres humanos. En la segunda fase, Arendt ya ha transitado por dos nuevos campos: el problema de Eichmann y el descubrimiento político de las revoluciones modernas. En este sentido, Arendt se centra en la ausencia de pensamiento en el mundo (Eichmann) y en la capacidad de nuestras facultades espirituales para hallar el significado de los acontecimientos políticos (revoluciones modernas). Por eso, algunos autores han destacado el carácter histórico y contemplativo de la segunda fase del juicio arentiano. La relación entre ambos modelos de juicio, no es, sin embargo tan sencilla y por ello se dedica este análisis a desentrañar la tensión que subyace en las dos fases del juicio arendtiano. Mi postura en este artículo es que la tensión entre estas dos fases refleja un problema fenomenológico de fondo, teñido por la postura postmetafísica de Hannah Arendt. En primer lugar, explico las implicaciones de los modelos de juicio existentes en el pensamiento de Arendt. A continuación, detallo la opción fenomenológica de Arendt desde la primacía que concede a la apariencia y su relación con lo político. Por último, hago explícito el problema del juicio arendtiano de la mano de Jean-Luc 
Marion, haciendo notar que la tensión entre el actor y el espectador es, en realidad, la tensión fenomenológica entre hermenéutica y tipología.

\section{Arendt y los dos modelos de juicio}

Coincido con Diana Taylor (2002) en que Ronald Beiner ha sido clave para establecer una división en las dos fases del juicio arendtiano. Dice Beiner:

En los textos anteriores al artículo de 1971, «El pensar y las reflexiones morales», el juicio se considera desde la óptica de la vita activa; en los textos posteriores, el juicio pasa a considerarse desde el punto de vista de la vida del espíritu (Beiner, 2003: 161).

Beiner (2003) se sirve del Postscriptum al capítulo dedicado al Pensamiento de la Vida del Espiritu y de las lecturas de Arendt sobre Kant para indicar que Kant es el interlocutor privilegiado de Arendt y que su reformulación del juicio conduce a una depolitización del mismo. De igual manera Richard Bernstein apuntala esta división con su Judging the Actor and the Spectator (1986) indicando la doble faceta del juicio arenditano en relación con las facultades humanas, aunque, como veremos, advierte que la tensión entre las dos fases no desaparece. Passerin D’Entrèves también señala estas dos etapas del juicio arendtiano:

una temprana en la que el juicio es la facultad de actores políticos que actúan en el ámbito público, y una posterior en la que es el privilegio de los espectadores no participantes, principalmente poetas e historiadores, que buscan comprender el significado del pasado y reconciliarnos con lo que ha sucedido (2014: 19).

La primera fase del juicio arendtiano está motivada por la necesidad de comprensión ante el desastre totalitario. Arendt decía acerca lo ocurrido:

Cuando lo imposible es hecho posible se torna en un mal absolutamente incastigable e imperdonable que ya no puede ser comprendido ni explicado por los motivos malignos del interés propio, la sordidez, el resentimiento, el ansia de poder y la cobardía. (1998: 368)

A pesar de tan abrumadora descripción, Arendt es consciente de la necesidad de reconciliación con el mundo, no para perdonar lo "imperdonable", sino para vivir admitiendo lo ocurrido. Así, en 1953, en Comprensión y Política decía: "comprender el 
totalitarismo no es indultar nada, sino reconciliarnos con un mundo en que tales cosas son posibles" (2005: 372). Pero comprender no es tan sencillo, ya que los totalitarismos han puesto en entredicho la facultad política por excelencia: el sentido común, que en Arendt es siempre una “idea regulativa” (Wellmer, 2001: 168; López, 2001: 113). "Los fenómenos totalitarios, que ya no pueden entenderse en términos del sentido común y que desafían a todas las reglas del juicio "normal" [...] son sólo los ejemplos más espectaculares del colapso de nuestra sabiduría común heredada” (Arendt, 2005: 381), la cual es sustituida por la lógica totalitaria, que, para Arendt, es ejercida mediante la coerción ideológica. Así, destruido el sentido común, Hannah Arendt busca en el juicio el tipo de cognición que pueda acompañar la acción de los hombres en medio del mundo. Si la acción implica comenzar, la reconciliación (comprensión) con el mundo es un requisito para iniciar nuevas acciones. Decía en Comprensión y política:

\begin{abstract}
Si la esencia de toda acción, y en particular de la política, es hacer un nuevo comienzo, entonces la comprensión se vuelve la otra cara de la acción, a saber: se vuelve esa forma de cognición, distinta de tantas otras, por la que los hombres que actúan (y no los hombres entregados a contemplar cierto curso progresivo o fatal de la Historia) pueden terminar por aceptar lo que irrevocablemente ha ocurrido y pueden reconciliarse con lo que insoslayablemente existe (2005: 391)
\end{abstract}

Arendt busca "esa forma de cognición” por la que se pueda aceptar lo que irrevocablemente ha ocurrido. En marzo de 1953 anota en su Diario Filosófico que la comprensión, entendida como reconciliación, "precede a toda acción y la posibilita" (2006: 321). Al año siguiente, prosigue la indagación sobre esta facultad cognitiva. Es en el texto llamado Sócrates donde Arendt presenta al phronimos, "hombre de entendimiento, cuyas intuiciones acerca de los asuntos humanos le cualifican para el liderazgo..." (2008: 41), distinguiendo entre la intuición política (phronesis) y el espíritu filosófico (nous) (2008: 70). Hannah Arendt está descubriendo, en estos primeros compases, los elementos de su primera teoría del juicio: la posibilidad de reconciliación, la anticipación a la acción y el recurso a la "intuición política” (phronesis).

En los escritos posteriores ampliará y profundizará en “esa forma de cognición” diferente del pensar filosófico. En La crisis en la cultura de 1960 Arendt, indica que el juicio es la facultad política por excelencia, distinguiendo lógica y facultad de juzgar. La lógica, en su individualidad es incapaz de salir del yo. En el caso de la facultad de juzgar, 
es la presencia de los otros la que le confiere validez. Para Hannah Arendt la filosofía política de Kant no se encuentra, como hasta entonces se había sugerido, en la Crítica de la Razón Práctica, donde se halla la dimensión legislativa de la razón, sino en la Crítica del Juicio, donde cree encontrar en el juicio de gusto el modelo para el juicio político. A Arendt "le interesa el Juicio en cuanto término medio entre teoría y práctica, en el sentido de que aplica las reglas teóricas al caso concreto [...] y si se carece de ellas, las busca" (Rivera de Rosales, 2005: 10). Las limitaciones del solipsismo habían sido evidenciadas por el aislamiento a que los seres humanos eran sometidos por los totalitarismos. La elaboración teórica privada oculta el posible sentido del mundo. Para comprender y remitirse al mundo se hace necesaria una facultad que rompa el círculo del aislamiento. Por eso Arendt afirma:

\begin{abstract}
Este modo de pensar amplio, por otra parte, que como juicio conoce la forma de trascender a sus propias limitaciones individuales, no puede funcionar en estricto aislamiento o soledad, sino que necesita la presencia de otros «en cuyo lugar» debe pensar, cuyos puntos de vista tomará en consideración y sin los cuales jamás tiene ocasión de entrar en actividad (1996: 233)
\end{abstract}

Que el juicio es una facultad de mundo y que su ámbito es la política lo evidencia el hecho de que Arendt considera fundamental el ejercicio de esa facultad en quienes se dedican a la actividad pública. Un dato relevante proviene de las reflexiones de Arendt a propósito de la Revolución Húngara de 1956, último exponente hasta la fecha del sistema de consejos, el cual es para ella la única alternativa de representación democrática al sistema multipartidista. A diferencia de éste último, representado en el Parlamento, el sistema de consejos nace de las acciones y demandas del pueblo y la elección de sus miembros está determinada por la integridad, coraje y "buen juicio" (Arendt, 2007b: 102).

Arendt sostiene que el juicio es la facultad política por excelencia, al hacer presente lo que está ausente y al tener en cuenta las perspectivas de los otros, gracias a la imaginación. El juicio de gusto kantiano sirve de modelo para el juicio político por la representatividad que genera la imaginación. Arendt, apoyándose en la interpretación de Jaspers (1995), extrae de la Crítica del Juicio sus implicaciones políticas y morales. La representatividad o "mentalidad amplia" (Arendt, 1996, p. 254) es lo que posibilita que 
en política podamos encontrar la tan ansiada "imparcialidad". El valor de un juicio y de una opinión no reside en su adecuación a un modelo (pensar especulativo) sino en el grado de imparcialidad, que deriva de ese pensar extendido, en el que las perspectivas de los otros son tenidas en cuenta. Dando un paso más, Arendt establece un vínculo desde Kant hasta Aristóteles para identificar este modo de pensar representativo con la phronesis como facultad política:

La capacidad de juicio es una habilidad política específica en el propio sentido denotado por Kant, es decir, como habilidad para ver cosas no sólo desde el punto de vista personal sino también según la perspectiva de todos los que estén presentes; incluso ese juicio puede ser una de las habilidades fundamentales del hombre como ser político, en la medida en que le permite orientarse en el ámbito público, en el mundo común... Los griegos llamaron Phronesis, discernimiento, a esa capacidad, y la consideraron la principal virtud o la excelencia del hombre de Estado, a diferencia de la sabiduría del filósofo (Arendt, 1996: 233).

Arendt parece haber solucionado la cuestión. El juicio permite, por una parte, recurrir a una capacidad no especulativa y alejarnos, así, de la filosofía. Se constituye, además, en la pluralidad de los otros, rompiendo el individualismo de aquel pensar solitario, donde tenían cabida las deducciones lógicas que implicaban las ideologías totalitarias. Gracias al juicio, Hannah Arendt piensa que ha devuelto el hombre al ámbito del sentido común.

En este primer momento, Arendt habría buscado en el juicio una herramienta práctica, previa al actuar político, íntimamente ligada con el actor. La búsqueda de comprensión y el rechazo a la dimensión contemplativa de la filosofía le llevó a renunciar al pensamiento puro y a proponer el ejercicio de la imaginación como solución hermenéutica. A esta primera etapa correspondían los escritos de comprensión de la década de los cincuenta, así como los ensayos Verdad y política y La crisis en la cultura. Aun perteneciendo a la primera etapa, Verdady Política de 1967 supone un punto de inflexión. Allí, Arendt ya se decanta por la narración de historias y el perspectivismo kantiano, pero todavía no ha desarrollado plenamente la articulación del segundo modelo. Será a partir de 1971 cuando, con Elpensar y las reflexiones morales, desarrolle su segunda teoría del juicio.

La principal diferencia entre las dos fases resulta, como decíamos, de considerar el juicio desde la perspectiva del actor (primera etapa) o desde la perspectiva del Rev. Filos., Aurora, Curitiba, v. 32, n. 55, p. 233-252, jan./abr. 2020 
espectador (segunda etapa). El escollo es, en ambos lugares, es cómo ejercer una facultad espiritual en relación con el mundo de apariencias (considerado desde la dimensión actuante de los hombres). Reconocer el segundo modelo del juicio conlleva asumir que el significado de los acontecimientos históricos pueda trascender las fronteras del tiempo. Es decir, que el significado de la apariencia en el mundo (y el acontecimiento histórico se constituye para ella como nuevo aparecer), dado por el ejercicio del juicio, pueda convertirse en fuente de significado más allá de su acontecer en un momento dado. En palabras de Passerin D’Entreves nos encontramos ante la "ejemplaridad histórica":

Para Arendt, esta noción de validez ejemplar no se limita a los objetos estéticos ni a los individuos que ejemplifican ciertas virtudes. Más bien, ella quiere extender esta noción a eventos en el pasado que tienen un significado más allá de lo que sucedió, es decir, a eventos que podrían ser vistos como ejemplares para quienes vinieron después. Es aquí donde el juicio estético se une al juicio retrospectivo del historiador (2000: 251).

En Verdady Política Arendt había reconocido la centralidad de la verdad factual contraponiéndola a la verdad teorética, propia de la contemplación. El problema, apuntado allí, era preservar la verdad factual para las generaciones futuras. De ahí el recurso a la narración. En Comprensión y política había ligado la imaginación a la posibilidad de comprender, pero sin ir más allá del "corazón comprensivo” (2005: 392). Es la segunda teoría del juicio la que procura la hilazón de ambas cuestiones de la mano de la relevancia dada al "acontecimiento": la verdad factual de un acontecimiento se convierte en fuente de significado político cuando la imaginación es capaz de dotarlo de ejemplaridad. A propósito de la Revolución Francesa, dice Arendt:

Pues ese acontecimiento es demasiado grandioso, se halla tan estrechamente ligado al interés de la humanidad, está de tal forma diseminado por todas partes a causa de su influencia sobre el mundo, como para no ser rememorado por los pueblos en cualquier ocasión propicia y evocado en orden a la repetición de nuevas tentativas de esa índole [...] (2003: 89).

La ejemplaridad salva la verdad del acontecimiento. El sentido historicista había provocado que "la verdad no correspondía ni se refería a los ciudadanos, entre los cuales sólo podía existir una multitud de opiniones, ni tampoco a los nacionales, cuyo sentido de la verdad estaba limitado por su propia historia y experiencia nacional" 
(Arendt, 1988: 55). Reservar el ejercicio del juicio al tribunal de la historia había privado a la verdad factual de su significado en el tiempo presente del mundo. Por ello, Arendt rechaza la postura de Hegel, desde quien "el pensamiento convirtió en histórico todo lo que había sido político” (Arendt, 1988: 69). Por eso es Kant y no Hegel, el interlocutor privilegiado de la segunda etapa del juicio:

O bien decimos con Hegel: Die Weltgeschichte ist das Weltgericht (la historia del mundo es el tribunal del mundo) dejando el juicio último al éxito, o bien afirmamos, con Kant, la autonomía del espíritu humano y su independencia potencial de las cosas como son o como han llegado a ser (Arendt, 2003: 18).

De esta manera, la segunda teoría del juicio arendtiano no está desligada de la política. La insistencia de Ronald Beiner en el carácter contemplativo de la segunda fase de Arendt pasa por alto la crítica de Arendt al pensamiento historicista de Hegel. El análisis de las revoluciones modernas indica que el sentido de las acciones individuales no puede esperar al final de la historia: "En la solución hegeliana, las acciones individuales permanecen tan carentes de sentido como antes, mientras que su proceso considerado en su totalidad revela una verdad que trasciende la esfera de los asuntos humanos" (Arendt, 2005: 518). Por eso, Arendt cree que el juicio reflexionante de Kant tiene la salida al problema. Como indica Sheyla Benhabib: "su último párrafo al Postscriptum pasa de la ética al problema de la historia. Ella tiene la intención de negar el derecho de la historia de ser la máxima jueza, "Die Weltgeschichte ist das Weltgericht" (Hegel), sin negar la importancia de la historia” (2006: 235).

La doble fase del juicio en Arendt nos lleva entonces al núcleo de un problema de índole fenomenológica: cómo encontrar un significado a lo que "aparece" en el mundo sin esperar que el tribunal de la historia revele la verdad de las acciones particulares.

\section{La fenomenología postmetafísica de la apariencia}

El problema del aparecer se presenta en el pensamiento "decididamente postmetafísico" (Wellmer, 1998: 75) de Arendt. En efecto, ella misma reconoce su 
adscripción a las filas de todos aquellos que han procurado desmantelar el edificio de la metafísica. Como indicaba en la La Vida del Espiritu:

Me he incorporado claramente a las filas de aquellos que desde hace ya algún tiempo se esfuerzan por desmontar la metafísica y la filosofía, con todas sus categorías, tal y como las hemos conocido desde sus comienzos en Grecia hasta nuestros días (2002: 242)

El carácter postmetafísico del pensamiento de Arendt se comprende desde su aceptación de la ruptura de la tradición y la consiguiente apuesta por un pensamiento sin barandillas (denken obne Geländer). Esta apuesta cobra carta de naturaleza al constatar que los totalitarismos "han hecho explotar, bien claramente, nuestras categorías de pensamiento político y nuestros patrones de juicio moral" (Arendt, 2005: 374). Por ello, la presentación de un Kant "post-metafísico" (López, 2001: 106) implica "ejercer el juicio práctico no como una forma de subordinar a un individuo a una regla existente, sino como un acto espontáneo y creativo, aunque situado en un entorno de crítica pública" (Burdon, 2015: 232). Arendt busca esta creatividad postmetafísica no sólo porque los totalitarismos han reventado nuestros patrones de juicio moral, sino porque descubre en la época moderna una doble ruptura: la ruptura ser / apariencia y la ruptura ser / pensar. Ambas posturas son el trasfondo de su postura postmetafísica.

La postmetafísica de Arendt se revela de manera clara al final de su vida cuando, al constatar que los seres humanos vivimos en un mundo de apariencias, señala la "falacia metafísica" (Arendt, 2002: 38) de la superioridad de una causa (ser) sobre el efecto (apariencia), denunciando el hecho de que haya que atribuir a la causa (sea esta el Ser o la Verdad) un "rango de realidad más elevado que el efecto" (Arendt, 2002: 38). De esta manera Arendt sale al paso de la sospecha moderna cartesiana respecto a las apariencias. En La Condición Humana indicaba que "por el contrario, este Ser es tremendamente activo y vigoroso: crea sus propias apariencias, aunque dichas apariencias son engañosas" (1993: 304). Arendt señala así que la falacia metafísica se nutre de la ruptura moderna entre ser y apariencia. Su postura postmetafísica rechaza tal ruptura, apostando por la coincidencia fenomenológica entre ser y apariencia: "en este mundo al que llegamos procedentes de ninguna parte y que abandonamos con idéntico 
destino ser y apariencia coinciden” (Arendt, 2002: 31). Arendt asume el postulado fenomenológico "cuánto hay de apariencia, tanto hay de ser" (Soviel Schein - soviel Sein) en el que tanto Husserl como Heidegger coinciden ${ }^{1}$. Aunque Heidegger indica en Introducción a la Metafísica (2001) que dicha unidad se perdió hace tiempo, Arendt afirma con rotundidad la "supremacía de la apariencia" (2002: 37). De esta manera, el carácter postmetafísico del juicio en Arendt (Pía Lara, 2007) implica aceptar esta suficiencia de la apariencia. Como indica Richard Bernstein: “juzgar, aunque es una actividad mental de la vida de la mente, nunca abandona el mundo de las apariencias" (1986: 236).

En la "Introducción" a La Vida del Espiritu recogía lo que había anticipado en El pensar y las reflexiones morales, reconociendo que Dios, la metafísica, la filosofía e incluso el positivismo han muerto. Son "muertes modernas" (Arendt, 1995: 113) que constituyen un "supuesto común no examinado de casi todo el mundo" (Arendt, 2002: 22) y que condensan la segunda ruptura moderna: la ruptura ser - pensar:

La unidad de pensar y ser tenía por presupuesto la coincidencia preestablecida de essentia y existentia: todo lo pensable existe, asimismo, y todo lo existente, en razón de su cognoscibilidad, ha de ser asimismo racional. Esta unidad fue demolida por Kant... (Arendt, 2005: 209).

Partiendo de tal demolición, el individuo moderno quedaba a merced de sí mismo. Por ello, Arendt conecta el solipsismo del Dasein de Heidegger con el individuo absoluto de Schelling, a quien Arendt considera el verdadero iniciador de la filosofía contemporánea. Encontramos aquí la razón del rechazo de Arendt a esta ruptura. Si pensar está, siguiendo a Heidegger, "fuera de orden” (Arendt, 1995: 117) y si "cuando pienso me muevo fuera del mundo de las apariencias" (Arendt, 1995: 116), hay que superar esta ruptura o, al menos buscar una alternativa para poder ser fiel al mundo de las apariencias. Es lo que ocurre en el juicio.

Arendt pretende superar el solipsismo del pensar, el cual propicia un alejamiento del sentido común (Lobo, 2012: 119). Por ello, acudía, como veíamos, al pensar ampliado de Kant. Pero también quiere superar la invisibilidad del pensar, ya que no

${ }^{1}$ La cita se remonta a J. F. Herbart (1968) Haupstücke der Metaphysik (1806), Sämtliche Werke. Frankfurt am Main, Bd2: 187. En Husserl la encontramos en las Meditaciones Cartesianas, § 46 (Hua I: 133). En Heidegger aparece en los Prolegómenos para una historia del concepto de tiempo (GA, 20: 119, 189) y en Ser y Tiempo, §7 (2012: 36). 
permitiría un encuentro con el mundo de apariencias. Si algo rechaza de los filósofos es que, tratando de fundar el conocimiento sobre lo que ofrece y elabora el pensamiento, se ven abocados al fracaso. Del pensamiento no queda sino su ejercicio destructivo (fundamental para evitar caer en ideologías) y su invisibilidad. Pero esto no es aceptable para los acontecimientos que necesitan ser mostrados. Criticando a Descartes, Arendt decía que, efectivamente, el pensamiento puede apropiarse de lo que es real acontecimiento, un objeto..., pero no puede apropiarse de la "realidad de cada uno de ellos" (2002: 65-66). De igual modo, decía que "la realidad es diferente de la totalidad de los hechos y acontecimientos y es más que ellos, aunque esta totalidad es de cualquier modo imprevisible" (Arendt, 1996: 275).

El contacto con la realidad es insoslayable. Es más, la narración de los hechos, el contar historias nos referencia a la realidad. Si los héroes clásicos son los hombres de la apariencia (andres epiphaneis), los filósofos serían los “seres invisibles" (Arendt, 2002: 90). Dado que el interés de Arendt reside en la visibilidad del acontecimiento, la práctica del juicio y el contar historias establecen un puente con los héroes clásicos (que en definitiva son todos aquellos que inician acontecimientos). Arendt va a buscar esos "héroes clásicos", en el germen de cualquier acontecimiento que merezca ser recordado, incluso entre los acontecimientos de la modernidad (los padres fundadores de la revolución americana).

Ahora bien, hacer visible el acontecimiento no deja de ser "fenomenología": "un estudio, una reflexión, una filosofía feromelógiea no una filosofía que sigu método particular o que concierne a un objeto específico, sino un pensamiento que logra mostrar los fenómenos" (Grondin, 2018: 120). Desde esta postura entendemos que el juicio arendtiano, en sus dos modelos, trata de seguir la senda hermenéutica de la fenomenología. Por ello, no podemos reducir el juicio arendtiano a su segunda etapa (Fine, 2007), donde aparece mucho más definido, sino establecer las conexiones con los escritos hermenéuticos de los cincuenta, rastreando cómo Arendt trata de hacer visibles los fenómenos. 
Hacer visible los fenómenos implica entonces movilizar ciertas facultades espirituales que muestren el acontecimiento a la luz pública. Por ello, al adoptar el juicio, Arendt está tratando de hacer que la visibilidad sea un asunto público. Y aquí aparece el problema fenomenológico de la caracterización del acontecimiento. Es decir, ¿cómo nos podemos referir a un acontecimiento? ¿Basta identificarlo para comprenderlo? Arendt identificó el fenómeno político por excelencia del siglo XX: llamó totalitarismo a una nueva forma de gobierno que hace del terror su principio inspirador. Pero eso no significa que el fenómeno ya esté comprendido. Como indicaba: "si queremos estar en casa en esta tierra, [...], hemos de tomar parte en el interminable diálogo con la esencia del totalitarismo" (Arendt, 2005: 393). Por eso, al final de su vida indicaba que todo lo que había escrito era provisional (Hill, 1979) y que su única motivación había sido comprender. "Para mí lo esencial es comprender, yo tengo que comprender. Y escribir forma parte de ello, es parte del proceso de comprensión” (Arendt, 2005: 19) decía en la conocida entrevista realizada por Günther Gauss para la televisión alemana en 1964. No obstante, ante Gershom Scholem, un año antes, Arendt indicaba que comprender es "despachar el pasado" (2010: 32), lo cual implicaba emitir juicios y "rotundos" (2010: 32).

Así pues nos encontramos con una tensión fenomenológica en la juicio arendtiano: o bien aceptamos una espiral hermenéutica de la que desconocemos su fin, o bien aceptamos la caracterización tipológica (ejemplar) de un acontecimiento hecho visible.

\title{
Tipología o hermenéutica ¿Se puede elegir?
}

\begin{abstract}
A lo largo de todo el trabajo de Arendt, existe una profunda tensión entre actuar y pensar, entre la perspectiva de los actores que participan en el espectáculo humano y la de los espectadores que buscan comprender su significado. Ella nunca reconcilió esta tensión, aunque a veces se sugiere una posible reconciliación (Bernstein, 1986: 237)
\end{abstract}

Esta tensión es la que encontramos en las dos fases del juicio arendtiano, la tensión entre la acción y la contemplación o, en términos fenomenológicos, la tensión 
entre hermenéutica o tipología. La alternativa parece no tener solución a tenor de opción de Grondin, quien termina proponiendo una fenomenología "tachada" (2018: 119), al renunciar a la fijación de una hoja de ruta fenomenológica. En lo que sigue profundizaremos en esta tensión del juicio arendtiano, siguiendo de cerca a Jean-Luc Marion, fenomenólogo con quien Arendt comparte inesperadas semejanzas (García y Vinolo, 2019).

Jean-Luc Marion en Siendo dado (2008a) caracteriza el evento o acontecimiento desde tres aspectos: irrepetible, incausado, impredecible. El acontecimiento, no previsto, se caracteriza por el arribo con que adviene a nosotros. Según Vinolo el acontecimiento se presenta como "un rayo que se da en un cielo azul" (2012: 97). De esta manera el carácter incausado del acontecimiento elimina cualquier interferencia del sujeto. Marion distingue entre arribo y llegada. La llegada es algo previsto por el sujeto: "el objeto procede de la visión que lo prevé, en cuanto su esencia, su posibilidad (lógica o trascendental) son conocidas antes de su existencia y la permiten; [...]" (Marion, 2010: 288). El arribo, sin embargo excede la temporalidad que lo precede, no permite ser capturado por previas categorizaciones. El arribo no permite ser condicionado. Por ello, añade Marion: "[...]. El evento aparece desapareciendo, puesto que no se hace visible sino "con" y por lo tanto "después" de su surgimiento: [...] (2010: 289). También Arendt reservaba para el acontecimiento el significado del "después". El acento en el arribo y la exclusión de la preeminencia del sujeto se inscriben en la fenomenología de la donación. Marion renuncia a la posible constitución de objetos y apuesta por la donación de fenómenos, de tal manera que puede decir que lo que se da “instituye al adonado" (Marion, 2001: 54).

Hablar del acontecimiento como fenómeno en Marion implica hacer una pequeña aclaración. Marion distingue cuatro tipos de fenómenos. a) El fenómeno "pobre" (objetos lógicos y matemáticos) se da casi únicamente en la categoría del concepto. Son pobres en intuición. b) Los fenómenos de "derecho común" son los objetos construidos por las ciencias naturales y físicas. Se trata de fenómenos construidos en función de un concepto. Por eso no hay novedad ni imprevisibilidad. El 
producto se conoce antes de ser mostrado. En ellos hay una mayor intuición que en los fenómenos pobres, pero la donación se ve perjudicada por el conocimiento previo del objeto. c) Fenómenos "saturados". Son aquellos en los que la intuición desborda el concepto. Dice Marion que "la donación no solamente inviste por completo la manifestación, sino que también la sobrepasa y modifica sus características comunes" (2008a: 366). El fenómeno saturado no sigue el esquema cartesiano de la construcción del objeto (Vinolo, 2016). La primacía y la iniciativa han pasado del sujeto al fenómeno. Por ello, en la fenomenología de la donación ya no se habla de sujeto, sino del “adonado". Y aquí es donde Marion pretende continuar más allá de la metafísica: "lo que la metafísica descarta como una excepción (la paradoja saturada), la fenomenología lo adopta aquí como su norma -todo fenómeno se muestra en la medida (o en la desmedida) en que se da" (2008b: 368). Marion parte de Kant para indicar las categorías que se ven saturadas por ciertos fenómenos. Así distingue cuatro tipos de saturación (cantidad, cualidad, relación, modalidad) identificando cuatro tipos de fenómenos saturados: acontecimiento, ídolo, carne, ícono). De esta manera el acontecimiento es el fenómeno que satura la categoría de la cantidad. Marion añade, además, la existencia de fenómenos que desbordan las cuatro categorías. Llegamos así al cuarto tipo de fenómenos: d) son aquellos fenómenos conocidos como "revelación".

Decir que el acontecimiento es un "fenómeno saturado" nos lleva al núcleo del problema apuntado al principio. ¿Qué es la saturación: hermenéutica o tipología? La respuesta la encontramos en la propia evolución del pensamiento de Marion. El pensador francés simplifica la clasificación de fenómenos de Siendo dado presentando el núcleo del problema en un fragmento de Certitudes Negativas: "De manera que los fenómenos se manifiestan según su distinción entre objetos (cuya fenomenicidad se limita a las condiciones de posibilidad de nuestra experiencia) y eventos (cuya fenomenicidad no conoce límite alguno, salvo el del mostrarse por sí mismo)" (Marion, 2010: 276). Por eso, aunque los fenómenos saturados, en principio puedan parecer escasos, existe la posibilidad de que la hermenéutica "le podría aplicar, el Sujeto, a cualquiera (o a casi cualquiera, este es todo el problema) de los fenómenos. Así muy 
lejos de que se den fenómenos saturados, podrías recibir cualquier fenómeno como un fenómeno saturado" (Vinolo, 2019: 107). Es lo que Marion reconoce como "banalidad de la saturación" (Marion, 2005). Eso significa que: "Todo puede convertirse en un fenómeno saturado siempre y cuando la manera según la cual se da no se limite, tal como nos lo impone la cotidianidad del mundo técnico, a la objetidad unívoca." (Marion, 2012: 151). La saturación como hermenéutica implica reconocer que en la percepción (que puede ser objetal o saturada) se opta por no imponer significación alguna al fenómeno percibido. Es percepción saturada, la cual conlleva dejar que la donación pueda darse a la intuición sin restricciones. Por el contrario, la percepción objetal trata de limitar la intuición "puesto que sometemos inmediatamente lo que aparece a un signo (y, por ende, a un sentido, a una significación)" (Vinolo, 2019: 109).

Marion admite que los fenómenos pueden ser recibidos como saturados bajo las modalidades del evento, el ídolo y la carne. Sin embargo, en la modalidad del ícono se aleja de la saturación como hermenéutica y parece afianzar la tipología del fenómeno. En el ícono se pone en juego la contra-intencionalidad de la mirada del otro. Es decir, no puedo dejar de identificar al otro. En este sentido, el otro me impone éticamente su significación como Otro. "Aquí vemos que, sobre el caso preciso del ícono, Marion retrocede en la hermenéutica para regresar a tipologías fenomenales" (Vinolo, 2019: 113).

En Arendt asistimos a la misma tensión en relación con el acontecimiento. Mi lectura de la situación es que Arendt comenzó la indagación sobre el totalitarismo movida por motivos hermenéuticos. En este sentido, nos encontraríamos con la mirada fenomenológica tratando de dejar al margen el concepto. Por eso en un primer momento, Arendt rechaza la contemplación y apuesta por una hermenéutica continuada:

Comprender no tiene fin y no puede por tanto producir resultados definitivos. Comprender es el modo específicamente humano de estar vivo; pues toda persona individual necesita reconciliarse con un mundo al que nació como un extraño, y en el cual, en razón de la unicidad de su persona, sigue siendo por siempre un extraño. La comprensión comienza con el nacimiento y acaba con la muerte (2005: 372)

No es de extrañar la coincidencia con Marion en el carácter infinito de la hermenéutica. En Siendo dado, la hermenéutica es "[...] una hermenéutica sin fin en el 
tiempo: [...]." (Marion, 2008a: 370). La fenomenología de la donación, cuando entiende la saturación como hermenéutica y no impone ninguna condición al fenómeno, ha de aceptar que el proceso de significación no termine nunca. En su versión más pura, Arendt entiende así la comprensión.

Sin embargo, al transitar por el juicio kantiano y tratar de apresar los acontecimientos en su propio significado Arendt sucumbe por momentos a la tentación de imponer un significado, como vimos en el caso de la Revolución Francesa. De esta manera descubrimos en Arendt una fenomenología como hermenéutica en los primeros compases (sobre todo en Comprensión y política) y una fenomenología más próxima a la tipología al final de su vida (muy claro en las Conferencias sobre la filosofía política de Kant). La primera teoría del juicio, intentaba liberar las posibilidades de la acción en relación con el mundo (hermenéutica). La segunda teoría del juicio, ligada a la particular lectura de Kant, procura caracterizar los acontecimientos históricos de acuerdo a sus posibilidades narrativas. El hecho de pretender asignar una especie de significado al acontecimiento acerca su postura a la de la tipología, ya que los ejemplares de juicio se alinean así con el ejercicio objetivante.

Pero, ¿por qué la sombra de la tipología? Para Marion había un motivo fundamental para no perder de vista la tipología: los crímenes del nazismo. El crimen por excelencia, el asesinato, implicaba reconocer el rostro del Otro, reconocerlo como tal, incluso para poder asesinarlo. Hoy en día nadie duda de que los nazis sabían que estaban matando seres humanos. "El simple hecho que hayan querido borrar o justificar sus acciones es la prueba que veían la contra-intencionalidad sobre los rostros que mataron" (Vinolo, 2019: 113). La tipología arranca allí donde los hombres no pueden ser eliminados, donde la humanidad no puede ser instrumentalizada. Tal vez encontramos en Arendt la misma motivación. La incursión en la hermenéutica no podría darse indefinidamente si en algún momento deja de considerar la singularidad del rostro del Otro y el mundo común que compartimos. La tensión entre hermenéutica y tipología en el pensamiento de Arendt se mantuvo en toda su trayectoria y resulta sorprendente y aleccionador el hecho de que el paralelismo con 
Marion pueda llegar, incluso a los motivos por los que dicha tensión parece no resolverse. Concluimos, precisamente, con la vinculación que Arendt establece entre hermenéutica (comprensión), tipología (significado) y reconciliación con el mundo: "El resultado del comprender es el significado, que nosotros engendramos en el proceso mismo de vivir en tanto en cuanto tratamos de reconciliarnos con lo que hacemos y sufrimos" (2005: 373).

\section{Referencias}

ARENDT, H. Lo que quiero es comprender. Madrid: Trotta, 2010.

ARENDT, H. La promesa de la política. Barcelona: Paidós, 2008.

ARENDT, H. Responsabilidady juicio. Barcelona: Paidós, 2007a.

ARENDT, H. Karl Marx y la tradición del pensamiento político occidental. Madrid: Encuentro, 2007b.

ARENDT, H. Diario filosófico. Barcelona: Herder, 2006.

ARENDT, H. Ensayos de comprensión 1930-1954, Madrid: Caparrós, 2005.

ARENDT, H. Conferencias sobre la filosofía política de Kant. Barcelona: Paidós, 2003.

ARENDT, H. La vida del espiritu. Barcelona: Paidós, 2002.

ARENDT, H. Los orígenes del totalitarismo. Madrid: Taurus, 1998.

ARENDT, H. Entre el pasado y el futuro. Barcelona: Península, 1996.

ARENDT, H. De la historia a la acción. Barcelona: Paidós, 1995.

ARENDT, H. La condición humana. Barcelona: Paidós, 1993.

ARENDT, H. Sobre la revolución. Madrid: Alianza, 1988.

BEINER, R. Ensayo interpretativo. Hannah Arendt y la facultad de juzgar. BEINER, R. (ed.). Conferencias sobre la filosofía política de Kant. Barcelona: Paidós, 2003.

BENHABIB, S. Hannah Arendt and the Redemptive Power of Narrative. In: WILLIAMS, G. (ed.). Hannah Arendt. Critical Assessments of Leading Political Philosophers. New York: Routledge: 2006. v. 1, p. 326-347.

BERNSTEIN, R. Judging the Actor and the Spectator. Philosophical Profiles, p. 221-238, 1986. 
BURDON, P. D. Hannah Arendt: on judgment and responsibility. Griffith Law Review, v. 24, n. 2, p. 221-243, 2015. https://doi.org/10.1080/10383441.2015.1058215.

DENNENY, M. The Privilege of Ourselves: Hannah Arendt on Judgment. In: HILL, M. A. (ed.). Hannah Arendt. The Recovery of the Public World. New York: St. Martin's, 1979.

FINE, R. Judgment and the reification of the faculties. A reconstructive reading of Arendt's Life of Mind. Philosophy and social criticism, v. 34, n. 1-2, p. 155-174, 2007.

GARCÍA, J.; VINOLO, S. Hannah Arendt y Jean-Luc Marion: el acontecimiento y los márgenes de la metafísica. Tópicos, Revista de Filosofía, n. 57, p. 207-234, 2019. https://doi.org/10.21555/top.v0i57.10602019.

GRAY, J. G. The Abyss of Freedom-and Hannah Arendt. In: HILL, M. A. (ed.). Hannah Arendt: The Recovery of the Public World. New York: St. Martin's, 1979.

GRONDIN, J. Le tournant herméneutique de la phénomenologie. Paris: Presses Universitaries de France, 2018.

HEIDEGGER, M. Introducción a la metafísica. Barcelona: Gedisa, 2001.

HILL, M. A. (comp.). Hannah Arendt. The Recovery of the Public World. New York: St Martin's Press, 1979.

JACOBITTI, S. Hannah Arendt and the Will. Political Theory, v. 16, p. 53-76, 1988.

JASPERS, K. Los grandes filósofos: Los fundadores del filosofar: Platón, Agustín, Kant. Madrid: Tecnos, 1995.

LOBO, M. F. Hannah Arendt y la pregunta por la relación entre el pensamiento y la acción. Buenos Aires: Biblos, 2012.

LÓPEZ, D. M. Hannah Arendt y la crítica de la facultad de juzgar. Tópicos, v. 9, p. 105-128, 2001.

MARION, J. L. La rigueur des choses, entretiens avec Dan Arbib. Paris: Flammarion, 2012.

MARION, J. L. Certitudes négatives. Paris: Grasset, 2010.

MARION, J. L. Siendo dado: Ensayo para una fenomenología de la donación. Madrid: Síntesis, 2008a.

MARION, J. L. Sobre la ontología gris de Descartes. Ciencia cartesiana y saber aristotélico en las Regulae, Madrid: Escolar y Mayo, 2008b.

MARION, J. L. Le visible et le révélé. Paris: Cerf, 2005.

MARION, J. L. De surcroît, Études sur les phénomènes saturés. Paris: PUF, 2001.

PASSERIN D'ENTREVES, M. Hannah Arendt. ZALTA, E. N. (ed.). The Stanford Encyclopedia of Philosophy. Stanford: Stanford University, 2014. 
PASSERIN D'ENTREVES, M. Arendt's theory of judgement. In: VILLA, D. (ed.). The Cambridge Companion of Hannah Arendt, New York: Cambridge University Press, 200. p. 245-261.

PÍA LARA, M. Narrating Evil. A postmetaphysical theory of reflective judgment. New York: Columbia University Press, 2007.

RIVERA DE ROSALES, J. Kant y Hannah Arendt. La comunidad del juicio reflexionante. Ideas y Valores, v. 54, p. 1-29, 2005.

STEINBERGER, P. J. Hannah Arendt on Judgment. American Journal of Political Science, v. 34, n. 3, p. 803-821, 1990.

TAYLOR, D. Hannah Arendt on Judgement: Thinking for Politics. International Journal of Philosophical Studies, v. 10, n. 2, p. 151-169, 2002.

VINOLO, S. Dieu n'a que faire de l'être - introduction à l'oeuvre de Jean-Luc Marion. Paris: Germina, 2012.

VINOLO, S. La tentation moderne de Jean-Luc Marion: le scandale de la saturation. Dialogue, v. 55, p. 343-352, 2016.

VINOLO, S. La fenomenología de la donación como relevo de la metafísica. Quito: Pontificia Universidad Católica de Ecuador, 2019.

WELLMER, A. Hannah Arendt on judgment. The unwritten doctrine of reason. BEINER, R.; NEDELSKY, J. (eds.). Judgment, Imagination, and Politics. Lanham: Rowman \& Littlefield, 2001. p. 165-181.

WELLMER, A. Hannah Arendt: sobre la revolución. Areté, v. 10, n. 1, p. 67-100, 1998.

RECIBIDO: 07/03/2019

APROBADO: $19 / 10 / 2019$

RECEIVED: 03/07/2019

APPROVED: 10/19/2019 